\title{
ON ADELIC STRINGS
}

\author{
Branko DRAGOVICH円 \\ Institute of Physics, P.O.Box 57, 11001 Belgrade, \\ Yugoslavia \\ Steklov Mathematical Institute, Moscow, Russia
}

\begin{abstract}
New approach to $p$-adic and adelic strings, which takes into account that not only world sheet but also Minkowski space-time and string momenta can be $p$-adic and adelic, is formulated. $p$-Adic and adelic string amplitudes are considered within Feynman's path integral formalism. The adelic Veneziano amplitude is calculated. Some discreteness of string momenta is obtained. Also, adelic coupling constant is equal to unity.
\end{abstract}

1. Since ancient times, scientists have been interested in unification of human knowledge on Nature. Superstring theory, recently resulted in the M-theory, is the best modern candidate for "theory of everything". It unifies all fundamental interactions. Gravity, nonabelian gauge symmetry, supersymmetry and space-time dimension are the most significant predictions of string theory. Superstring theory is a synthesis of deep physical principles and modern mathematical methods. In spite of many significant achievements, there are a number of open questions. One of the main actual problems is the space-time structure at the Planck scale. To find an appropriate answer to this problem it seems unavoidable to extend the Riemannian geometry, which is the Archimedean one and related

\footnotetext{
${ }^{1}$ e-mail: dragovic@phy.bg.ac.yu
} 
to real numbers, with the non-Archimedean geometry described by $p$-adic numbers. The corresponding mathematical instrument suitable for unification of Archimedean and non-Archimedean geometries, as well as of real and $p$-adic numbers, is the space of adeles.

Recall that the field of rational numbers $Q$ plays an important role not only in mathematics but also in physics. All numerical results of measurements belong to $Q$. On $Q$ there is the usual $\left(|\cdot|_{\infty}\right)$ and $p$-adic $\left(|\cdot|_{p}\right)$ absolute value, where $p$ denotes a prime number. Completion of $Q$ with respect to $|\cdot|_{\infty}$ and $|\cdot|_{p}$ yields the field of real $\left(R \equiv Q_{\infty}\right)$ and $p$-adic $\left(Q_{p}\right)$ numbers, respectively. Many properties of $p$-adic numbers and their functions [1] are very different in comparison with real numbers and classical analysis. Any $x \in Q_{p}$ can be presented in the form

$$
x=p^{\nu}\left(x_{0}+x_{1} p+x_{2} p^{2}+\cdots\right), \quad x_{0} \neq 0, \quad \nu \in Z,
$$

where $x_{i} \in\{0,1, \ldots, p-1\}$.

An adele $a$ [2] is an infinite sequence

$$
a=\left(a_{\infty}, a_{2}, a_{3}, \ldots, a_{p}, \ldots\right)
$$

where $a_{\infty} \in R$ and $a_{p} \in Q_{p}$ with restriction that $a_{p} \in Z_{p}=$ $\left\{x \in Q_{p}: x=x_{0}+x_{1} p+x_{2} p^{2}+\cdots\right\}$ for all but a finite set $S$ of primes $p$. The set of all adeles $A$ may be given in the form

$$
A=\bigcup_{S} \mathcal{A}(S), \quad \mathcal{A}(S)=R \times \prod_{p \in S} Q_{p} \times \prod_{p \notin S} Z_{p} .
$$

$A$ has the structure of a topological ring.

2. Since 1987, $p$-adic numbers and adeles have been successfully used in many branches of theoretical and mathematical physics (for a review, see, e.g. [3], [4], and [5]). 
First applications were just in string theory. It was suggested [6] a hypothesis on the possible emergence of a nonArchimedean geometry at the Planck scale and initiated the corresponding string theory over $Q_{p}$ and the Galois fields. It was also proposed generalization of the usual Veneziano and the Virasoro-Shapiro amplitude using multiplicative characters over various number fields. In [7], the Veneziano and the Virasoro-Shapiro $p$-adic string amplitudes are postulated as the Gel'fand-Graev beta functions, which are convolutions of the real-valued characters on the $p$-adic fields. After that it was put forward an interesting formula [8]: the product of the Veneziano amplitude and its $p$-adic counterparts is equal to unity. In other words, the Veneziano amplitude can be presented as the infinite product of inverses of $p$-adic amplitudes. The similar property holds for the Virasoro-Shapiro amplitude. The product of an ordinary string amplitude and the corresponding $p$ adic counterparts is usually called adelic string amplitude. However, such adelic string amplitudes must be regularized [9] and they are not equal to a constant in a more complex case. This situation has been an obstacle to further progress in application of $p$-adic numbers in string theory. It seems to be a consequence of restricted employment of $p$-adic numbers in string amplitudes. In fact, a general feature of all these investigations was that only world sheet may be treated $p$-adically, while target space-time and momenta should be described by real numbers.

3. According to successful formulation of $p$-adic [10] and adelic [11] quantum mechanics, approach to $p$-adic and adelic string amplitudes should be modified. Here we give a new proposal to $p$-adic and adelic string amplitudes based on the following general assumptions:

(i) space-time and matter are adelic at the Planck (string) 
scale,

(ii) the Feynman path integral is an essential constituent of quantum theory,

(iii) ordinary classical and quantum theories are effective limits of the adelic ones.

Thus, a string is adelic, and has real and all $p$-adic faces simultaneously. We use term real ( $p$-adic) string when we consider as dominant its real ( $p$-adic) characteristics. In addition to the adelic Minkowski space-time, string world sheet and momenta are also adelic. However, string amplitude remains complex-valued in all these cases.

Recall that quantum amplitudes defined by means of path integral may be symbolically presented as

$$
A(K)=\int A(X) \chi\left(-\frac{1}{h} S[X]\right) \mathcal{D} X,
$$

where $K$ and $X$ denote classical momenta and configuration space, respectively. $\chi(a)$ is an additive character, $S[X]$ is a classical action and $h$ is the Planck constant.

4. In the sequal, we briefly consider simple $p$-adic and adelic bosonic string amplitudes based on the functional integral (4). As is known [12, the scattering of two real bosonic strings in 26-dimensional space-time at the tree level can be described in terms of the path integral in 2dimensional quantum field theory formalism as follows:

$$
\begin{gathered}
A_{\infty}\left(k_{1}, \ldots, k_{4}\right)=g_{\infty}^{2} \int \mathcal{D} X \exp \left(\frac{2 \pi i}{h} S_{0}[X]\right) \\
\times \prod_{j=1}^{4} \int d^{2} \sigma_{j} \exp \left(\frac{2 \pi i}{h} k_{\mu}^{(j)} X^{\mu}\left(\sigma_{j}, \tau_{j}\right)\right),
\end{gathered}
$$

where $\mathcal{D} X=\mathcal{D} X^{0}(\sigma, \tau) \mathcal{D} X^{1}(\sigma, \tau) \ldots \mathcal{D} X^{25}(\sigma, \tau), \quad d^{2} \sigma_{j}=$ $d \sigma_{j} d \tau_{j}$ and

$$
S_{0}[X]=-\frac{T}{2} \int d^{2} \sigma \partial_{\alpha} X^{\mu} \partial^{\alpha} X_{\mu}
$$


with $\alpha=0,1$ and $\mu=0,1, \ldots, 25$. Using the usual procedure [12] one can obtain the crossing symmetric Veneziano amplitude

$$
A_{\infty}\left(k_{1}, \ldots, k_{4}\right)=g_{\infty}^{2} \int_{R}|x|_{\infty}^{k_{1} k_{2}}|1-x|_{\infty}^{k_{2} k_{3}} d x
$$

and similarly the Virasoro-Shapiro one for closed bosonic strings.

As $p$-adic Veneziano amplitude, it was postulated [7] $p$ adic analogue of (7), i.e.

$$
A_{p}\left(k_{1}, \ldots, k_{4}\right)=g_{p}^{2} \int_{Q_{p}}|x|_{p}^{k_{1} k_{2}}|1-x|_{p}^{k_{2} k_{3}} d x
$$

where only the string world sheet (parametrized by $x$ ) is p-adic. Expressions (7) and (8) are Gel'fand-Graev beta functions on $R$ and $Q_{p}$, respectively. However, (8) cannot be derived from $p$-adic analogue of (5) and one may doubt validity of (8) as a $p$-adic string amplitude. Therefore, we take $p$-adic analogue of (5), i.e.

$$
\begin{gathered}
A_{p}\left(k_{1}, \ldots, k_{4}\right)=g_{p}^{2} \int \mathcal{D} X \chi_{p}\left(-\frac{1}{h} S_{0}[X]\right) \\
\times \prod_{j=1}^{4} \int d^{2} \sigma_{j} \chi_{p}\left(-\frac{1}{h} k_{\mu}^{(j)} X^{\mu}\left(\sigma_{j}, \tau_{j}\right)\right),
\end{gathered}
$$

to be $p$-adic string amplitude, where $\chi_{p}(u)=\exp \left(2 \pi i\{u\}_{p}\right)$ is $p$-adic additive character and $\{u\}_{p}$ is the fractional part of $u \in Q_{p}$. In (9), all space-time coordinates $X_{\mu}$, momenta $k_{i}$ and world sheet $(\sigma, \tau)$ are $p$-adic.

Evaluation of (9), in an analogous way to the real case, leads to

$$
A_{p}\left(k_{1}, \ldots, k_{4}\right)=g_{p}^{2} \prod_{j=1}^{4} \int d^{2} \sigma_{j}
$$




$$
\times \chi_{p}\left(\frac{\sqrt{-1}}{2 h T} \sum_{i<j} k_{i} k_{j} \log \left(\left(\sigma_{i}-\sigma_{j}\right)^{2}+\left(\tau_{i}-\tau_{j}\right)^{2}\right)\right) .
$$

Since $p$-adic character $\chi_{p}$ is complex-valued and logarithmic function is $p$-adic valued, amplitude (10) cannot be reduced to (8). $p$-Adic logarithmic function is

$$
\log x=\sum_{n=1}^{\infty}(-1)^{n+1} \frac{(x-1)^{n}}{n}, \quad|x-1|_{p}<1 .
$$

Due to (10) and (11) one obtains the system of equations

$$
\left(\sigma_{i}-\sigma_{j}\right)^{2}+\left(\tau_{i}-\tau_{j}\right)^{2} \in 1+p Z_{p}, \quad(i<j), i, j=1, \ldots, 4,
$$

which determines possible $p$-adic values of the world sheet parameters $\sigma_{i}$ and $\tau_{i}$. Since (10) can be rewritten as

$$
\begin{aligned}
& A_{p}\left(k_{1}, \ldots, k_{4}\right)=g_{p}^{2} \prod_{j=1}^{4} \int d^{2} \sigma_{j} \chi_{p}(\sqrt{-1} \\
& \left.\times \log \prod_{i<j}\left(\left(\sigma_{i}-\sigma_{j}\right)^{2}+\left(\tau_{i}-\tau_{j}\right)^{2}\right)^{\frac{k_{i} k_{j}}{2 h T}}\right)
\end{aligned}
$$

it has to be also satisfied

$$
\prod_{i<j}\left(\left(\sigma_{i}-\sigma_{j}\right)^{2}+\left(\tau_{i}-\tau_{j}\right)^{2}\right)^{\frac{k_{i} k_{j}}{2 h T}} \in 1+p Z_{p}
$$

Combining (12) and (14) one gets

$$
\prod_{i<j}\left(1+p Z_{p}\right)^{\frac{k_{i} k_{j}}{2 h T}} \in 1+p Z_{p}
$$

what is satisfied if

$$
\left|\frac{k_{i} k_{j}}{2 h T}\right|_{p} \leq 1
$$


Since in experiments $k_{i}, k_{j} \in Q$ and (17) has place for every $p$, it follows that string momenta have discrete values in units $h=T=1$.

Amplitude (14) becomes

$$
A_{p}\left(k_{1}, \ldots, k_{4}\right)=g_{p}^{2} \prod_{j=1}^{4} \int d^{2} \sigma_{j},
$$

because $\chi_{p}(\sqrt{-1} \log u)=1$.

5. Adelic string amplitude is product of real and all $p$ adic amplitudes, i.e.

$$
A_{A}\left(k_{1}, \ldots, k_{4}\right)=A_{\infty}\left(k_{1}, \ldots, k_{4}\right) \prod_{p} A_{p}\left(k_{1}, \ldots, k_{4}\right) .
$$

In the case of the Veneziano amplitude and $\left(\sigma_{i}, \tau_{j}\right) \in \mathcal{A}(S) \times$ $\mathcal{A}(S)$, where $\mathcal{A}(S)$ is defined in (3), we have

$$
\begin{gathered}
A_{A}\left(k_{1}, \ldots, k_{4}\right)=g_{\infty}^{2} \int_{R}|x|_{\infty}^{k_{1} k_{2}}|1-x|_{\infty}^{k_{2} k_{3}} d x \\
\times \prod_{p \in S} g_{p}^{2} \prod_{j=1}^{4} \int d^{2} \sigma_{j} \times \prod_{p \notin S} g_{p}^{2} .
\end{gathered}
$$

There is a sense to take adelic coupling constant as

$$
g_{A}^{2}=|g|_{\infty}^{2} \prod_{p}|g|_{p}^{2}=1, \quad 0 \neq g \in Q
$$

At the end, it follows that $p$-adic effects in the adelic Veneziano amplitude induce discreteness of string momenta and contribute to an effective coupling constant in the form

$$
g_{\text {ef }}^{2}=g_{A}^{2} \prod_{p \in S} \prod_{j=1}^{4} \int d^{2} \sigma_{j} \geq 1 .
$$




\section{Acknowledgments}

The author wishes to thank A.I. Studenikin and the Organizing Committee of the 9th Lomonosov Conference on Elementary Particle Physics for invitation to participate and give a talk.

\section{References}

[1] W.H.Schikhof, Ultrametric Calculus: an introduction to p-adic analysis, (Cambridge University Press, Cambridge) 1984 .

[2] I.M.Gel'fand, M.I.Graev and I.I.Piatetskii-Shapiro, Representation Theory and Automorphic Functions, (Nauka, Moscow) 1966.

[3] L.Brekke and P.G.O.Freund, Phys. Rep. 233, 1 (1993).

[4] V.S.Vladimirov, I.V.Volovich and E.I.Zelenov, $p$-Adic Analysis and Mathematical Physics, (World Scientific, Singapore) 1994.

[5] A.Khrennikov, p-Adic Distributions in Mathematical Physics, (Kluwer Acad. Publishers, Dordrecht) 1994.

[6] I.V.Volovich, Class. Quantum Grav. 4, L83 (1987).

[7] P.G.O.Freund and M.Olson, Phys. Lett. B 199, 186 (1987).

[8] P.G.O.Freund and E.Witten, Phys. Lett. B 199, 191 (1987). 
[9] I.Ya.Aref'eva, B.Dragovich and I.V.Volovich, Phys. Lett. B 209, 445 (1988).

[10] V.S.Vladimirov and I.V.Volovich, Commun. Math. Phys. 123, 659 (1989).

[11] B.Dragovich, Teor. Mat. Fizika 101, 1404 (1994).

[12] M.B.Green, J.H.Schwarz and E.Witten, Superstring Theory, (Cambridge University Press, Cambridge) 1984. 